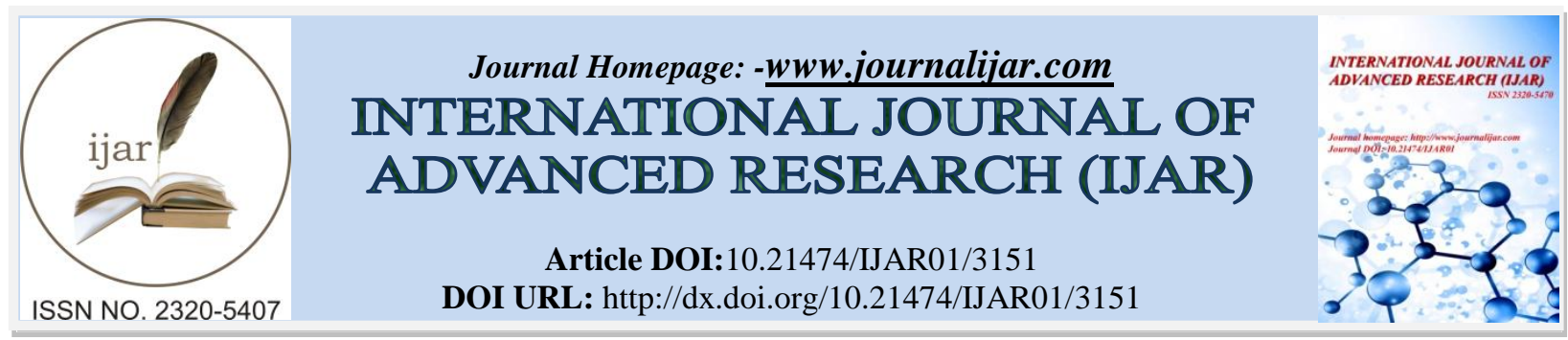

RESEARCH ARTICLE

\title{
A PROXIMAL LEFT MAIN BRONCHIAL INJURY ; A MANAGEMENT DILEMMA
}

Yasser Aljehani and Elham Al Qarniand Sarah Al Arafah.

Division of Thoracic Surgery, Department of Surgery. King Fahad Hospital of the University. University of Dammam. Dammam, Saudi Arabia.

\section{Manuscript Info}

Manuscript History

Final Accepted: 10 January 2017

Published: February 2017

Key words:-

Tracheobronchial injury, left main
Received: 15 December 2016 bronchus injury.

\begin{abstract}
Tracheobronchial injuries are rare injuries that occur in less than $1 \%$ of patients following chest trauma. It should be considered in almost all patient presenting with blunt chest trauma. The diagnosis initially depends on careful history and physical examination in addition to high index of suspicion and accurate interpretation of radiological findings. The prompt recognition of the injury, skillful airway management and early treatment greatly reduce morbidity and increase the chances of restoring normal pulmonary function. In case of proximal left main bronchus injury ideally it should be exposed via a right posterolateral thoracotomy. This will provide a good exposure to the left main-stem bronchus and the carina compared to approaching it thorough a left posterolateral thoracotomy where the overlying aortic arch will obscure the field and makes it difficult to repair. We present a case of proximal left main bronchus injury that underwent left posterolateral thoracotomy repair with all difficulties because the left lung was lacerated and single lung isolation was not tolerated.
\end{abstract}

Copy Right, IJAR, 2017,. All rights reserved.

\section{Introduction:-}

The first reported case of traumatic ruptured bronchus is attributed to Webb in 1848 following a postmortem on a man run over by a cart (1). Primary surgical repair was first successfully performed by Scannell in 1951 (2). Tracheobronchial disruption is an uncommon but life threatening injury associated with blunt thoracic trauma. Any injury to the trachea or major bronchi is commonly associated with high-speed motor vehicle accidents, In a large trauma autopsy series, $2 \%$ were found to have a tracheobronchial injury. Of those $81 \%$ died at the scene.

\section{Case presentation:-}

A 13 years old male referred from another hospital as a victim of road traffic accident. The patient was transferred from a peripheral hospital almost 24 hours after his injury. He was the driver and it was head on collision. Initial assessment reveled Glasgow coma scale was $8 \backslash 15$ for which he was intubated. His initial vital signs were normal and initial chest $\mathrm{x}$-ray showed bilateral hemopneumothorax and evidence of lung contusion for which bilateral chest tubes were inserted. His work up demonstrated the following injuries based on pan computed tomography scan; head was unremarkable, his chest showed: intact mediastinal major vasculature with no definitive extravasation. Presence of pneumomediastinum extending through the neck region. Bilateral lung contusion and laceration more prominent at the left upper lobe. Right upper lobe consolidation collapse. Bilateral pneumothorax were demonstrated. There was a bilateral subcutaneous emphysema more extensive in the left side and associated with

Corresponding Author:-Yasser Aljehani.

Address:-Division of Thoracic Surgery, Department of Surgery. King Fahad Hospital of the 
underlying rib fracture from the first to fourth ribs. Thin paper like appearance of the left bronchial away from the carina about $2 \mathrm{~cm}$ likely represents bronchial injury (figure1). Abdomen showed a Grade 2 splenic injury. The patient drained from left chest tube around $>800 \mathrm{cc}$ in 4 hours. The presence of significant left hemothorax due to possible lung laceration dictated exploration (figure2). Prior to exploration, flexible bronchoscopy was done and demonstrated a proximal left main bronchus injury with complete separation of both ends around $<2 \mathrm{~cm}$ from the carina. Approaching the right chest through right posterolateral thoracotomy would dictated isolating the right lung. The expected damage to the left lung and significant hemothorax would not be a good option for intraoperative maintenance of oxygenation. The decision was taken to explore the left chest through left posterolateral thoracotomy. Upon access, hemothorax and clots were evacuated and a left upper lobe laceration around $8 \mathrm{~cm}$ identified as the source of the bleeding. The examination of mediastinum showed the distal end of the left main bronchus and the proximal end was embedded under the aortic arch. Mediastinal dissection cleared both ends and primary anastomosis was done first by the posterior membranous layer by continuous running suture PDS $4 \backslash 0$ and followed by the anterior layer by an interrupted suture with the same type. The patient tolerated the procedure well his post op bronchoscopy showed patent left main bronchus. Unfortunately, the patient went into sepsis, multi-organ failure and passed away after 6 weeks from this procedure.

Figure 1:-

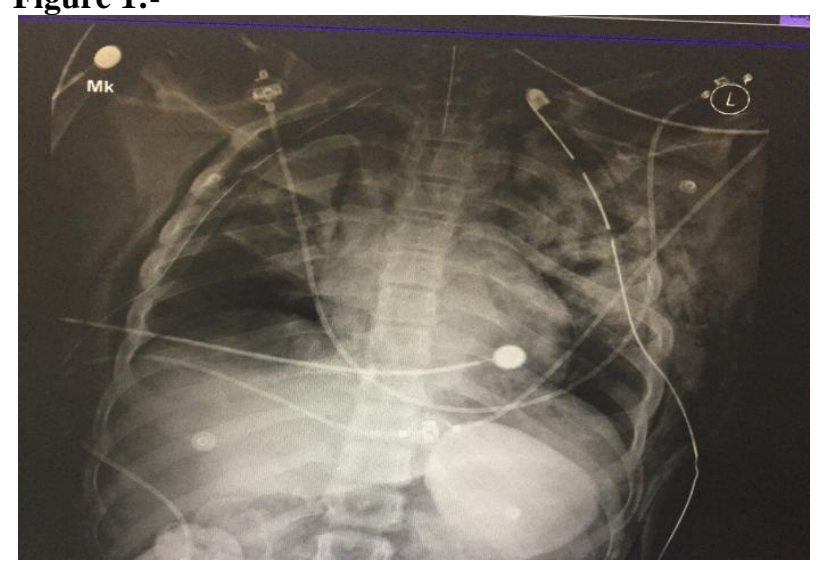

Figure 2:-

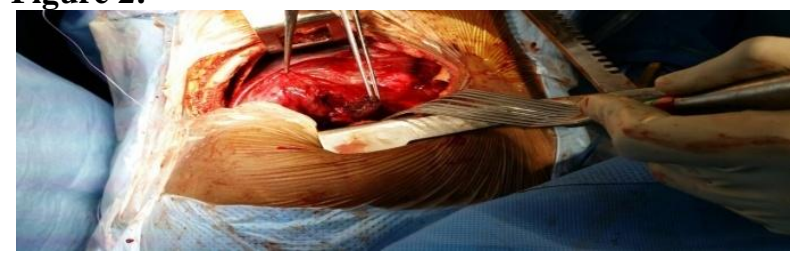

Figure 2:-

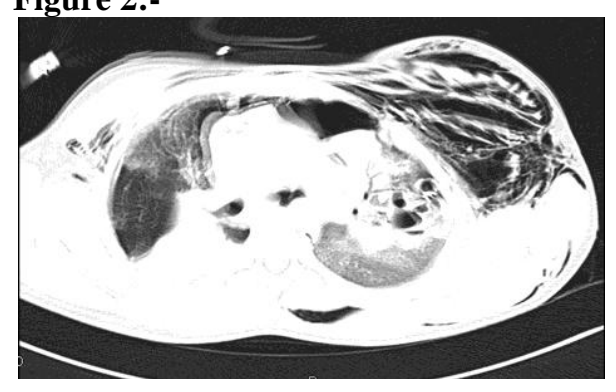




\section{Discussion:-}

Most tracheobronchial injuries (TBI) occurred within $2 \mathrm{~cm}$ of the carina. Injury to the right main bronchus is more common and diagnosed earlier. This is thought to be due to the fact that the left main bronchus is protected by the aorta. The median days until diagnosis for left sided injury was 30 days. The presentation for late diagnosis is often persistent pneumothorax. Historically, the outcome for left sided injury is more favorable than the right side, with a mortality rate of $8 \%$ compared with $16 \%$ (3). TBI usually varies from simple minor tear to avulsion or rupture at different locations. Road traffic accidents accounted for the majority of patients (59\%), followed by crush injuries (27\%) (4). The mortality from traumatic TBI has decreased from 36\% before 1950 and $30 \%$ in 1966 to $9 \%$ in 2001(5). The most common presenting signs of traumatic TBI were subcutaneous emphysema (43.5\%) and followed by pneumomediastinum (17.4\%) (6).It is associated with a high degree of lethality. The mechanism of injury is attributed to the following three hypotheses: First: direct chest trauma will lead to decrease the anterior posterior diameter of the thorax with increase in the transvers diameter. As the lungs remain always in contact with the chest wall because of the negative intra-pleural pressure, they stretch laterally and produce a traction force to the carina. Second: shearing of the bronchus from its points of origin near the carina, resulting in rapid deceleration. Third: the greatest wall tension generated within the trachea bronchial tree with increased air way pressures, during compression of the chest wall against a closed glottis is at the carina (3). The initial management should follows the Advance Trauma Life Support protocol. The outcome of non-operative management is generally worse than operative management in certain types of TBI. Surgical repair should be performed as soon as possible. If an injury is identified early, primary repair should be attempted. The operative approach differs depending on the location of the injury. Findings on chest x-ray can include pneumothorax, pneumomediastinum, subcutaneous emphysema and air surrounding deep cervical tissue. "Fallen lung sign" where the collapsed lung falls away from the mediastinum is not often seen but is specific to bronchial injury. Findings on CT are similar to those of chest $\mathrm{x}$-ray(3). The definitive diagnosis is made by bronchoscopy which is the gold standard. Early reconstruction depends on early diagnosis. If there is complete rupture the bronchoscopy should be used as a guide during ventilation. Cervical trachea injury is repaired via collar incision. Distal trachea, carina, and right main stem bronchus are approached through right posterolateral thoracotomy. The distal third of the trachea, carina and right main stem bronchus as well as the proximal left main stem bronchus is approached by a right thoracotomy. The distal left main bronchus will be better exposed via a left posterolateral thoracotomy. Debridement and end to end anastomosis must be attempted for significant tracheal and bronchial injury. Pneumonectomy should be avoided if possible. Lobectomy is performed if the injury is associated with lobar destruction. The mortality for those who underwent primary repair was lower than those who underwent resection of the injured bronchus and distal lung parenchyma (3\% vs 13\%) (3). Some patients cannot be approached conservatively therefore the most appropriate treatment is the surgical treatment as early as possible. The principles of surgery dictate the following: debridement of devitalized tissue including cartilage, end to end anastomosis, flexion of the neck to avoid anastomosis line tension and supporting the suture line with the local tissues as a flap (7). Left thoracotomy provides a good exposure to the distal left main-stem bronchus, distal part of aortic arch, descending thoracic aorta and proximal left subclavian artery. Nevertheless, it is hard to reach to the proximal left main stem bronchus, carina because of the overlying aortic arch and this is what we had to face in this case. The penetrating injuries in the chest are most probably accompanied by life threatening cardiovascular injuries. While median sternotomy ensures optimal access to the hearth and major vessels, it is less beneficial for trachea, carina and bronchi. Moreover, it does not provide appropriate exposure for the restoration of additional esophageal injuries (7). The severity of associated lung injury is taken into consideration regarding the approach and that what exactly what we had to experience. Massive hemothorax or massive air leakage are two indications for rapid surgical intervention in TBI (8). In delayed presentation; sequelae of stricture and stenosis (atelectasis or bronchiectasis) predominates. Fiber optic bronchoscopy is indicated and findings indicating injury includes clotted blood, edema, or erythema. However, these underestimate the real extent of the injury. In acute setting, surgical repair is the standard. Indications for surgery depend on clinical, radiological and endoscopic assessment, but clear indications are tension pneumothorax, bronchopleural fistula after drainage, increasing pneumomediastinum, or surgical emphysema (despite conservative measures), transmural tear $>2 \mathrm{~cm}$, and prolapsed esophageal wall into trachea or mediastinitis. In delay settings, granulation and fibrosis starts to fill the distal ruptured segment in around 3 weeks. Some authors advice to wait and control in sepsis of the stump before surgery (9). In our case the left lung was lacerated and the patient would not have tolerated single lung ventilation, isolating the right lung and depending on the left lung. In conclusion, diagnosis of airway injuries depends on high index of suspicion with adjunct diagnostic modalities. The principles of approach and surgical management is agreed upon but exceptional cases have to be managed depending on many factors including associated lung injury and tolerance to single lung isolation. 
1. Majdi Ibrahim, Hasan Sandogji, Abdallah Allam (2012) 'Avulsion of the right main bronchus due to blunt trauma', Journal of Emergency Medicine, Trauma and Acute Care , (), pp. 2 [Online]. Available at: http://www.qscience.com/doi/pdf/10.5339/jemtac.2012.5 (Accessed: 10 may 2012 ).

2. MagdiIbrahim ,MDHamdy D. El ayoty ,MD Hany ELdomiaty ,MD ( 2007) 'SURGICAL EXPERIENCE OF FRACTURE BRONCHUS', The Journal of Egyptian Society of Cardiothoracic Surgery , 15(1,2 ), pp. 134 [Online]. Available at: http://escts.net/upload/file/c09ad97eb691abc5055ab3edc84aa7c3.pdf (Accessed: Jule7 , 2007).

3. Ali Cheaito, MD, F.A.C.S, $\square$ AretiTillou, MD, Catherine Lewis, MD, and Henry Cryer, MD (2016) Traumatic bronchial injury, https://www.ncbi.nlm.nih.gov/pmc/articles/PMC5021783/: 2016 Sep 4.

4. Andy C. Kiser, MD, Sean M. O’Brien, MS, and Frank C. Detterbeck, MD (2001) 'Blunt Tracheobronchial Injuries: Treatment and Outcomes', THE ANNALS OF THORACIC SURGERY, 71(), pp. 2059-2065 [Online]. Available at: http://www.emergpa.net/wp/wp-content/uploads/2012/02/Ann-thorac-Surg-Blunt-trachealinjuries.pdf (Accessed: November 29, 2011).

5. Christos Prokakis, Efstratios N KoletsisEmail author, Panagiotis Dedeilias, FotiniFligou, KritonFilos and DimitriosDougenis (2014) 'Airway trauma: a review on epidemiology, mechanisms of injury, diagnosis and treatment', Journal of Cardiothoracic Surgery, (), pp. [Online]. Available at: https://cardiothoracicsurgery.biomedcentral.com/articles/10.1186/1749-8090-9-117 (Accessed: 30 June 2014).

6. Jung Joo Hwang, M.D., Young Jin Kim, M.D., Hyun Min Cho, M.D.,corresponding author and Tae Yeon Lee, M.D. (2013) 'Traumatic Tracheobronchial Injury: Delayed Diagnosis and Treatment Outcome', Korean J ThoracCardiovasc Surg., 2(46), pp. 197-201 [Online]. Available at: https://www.ncbi.nlm.nih.gov/pmc/articles/PMC3680605/ (Accessed: jun2013).

7. Tamer Altinok and Axilla Can (2014 Aug 26.) Management of Tracheobronchial Injuries, https://www.ncbi.nlm.nih.gov/pmc/articles/PMC4299837/: oct 2014.

8. Rahim Mahmodlou and NarimanSepehrvand (2015) 'Tracheobronchial injury due to blunt chest trauma', US National Library of Medicine National Institutes of Health, 2(5), pp. 116-118 [Online]. Available at: https://www.ncbi.nlm.nih.gov/pmc/articles/PMC4477389/ (Accessed: Apr-Jun 2015).

9. Yasser M. Aljehani, MD, SCC Surg, Mohamed H. Ahmad, MD, FFCTS, Omniah A. Alashgar, MBBS, Mahmoud H. Ashour, FRCS, FEBTCS. (2013) 'Delayed bronchial rupture repair A revisit to mucus basics', saudi medical journal , 34(2), pp. 195-198 [Online]. Available at: www.smj.org.sa/index.php/smj/article/download/592/365 (Accessed: 2013). 